\title{
Higher baseline serum adiponectin predicts better treatment remission in patients with generalized anxiety disorder treated with escitalopram
}

\author{
Zhongxia Shen ${ }^{1,2 \#}$, Feng Wang ${ }^{3 \#}$, Lijun Cui ${ }^{2}$, Lie Ren², Xiujuan Hong', Yonggui Yuan ${ }^{4}$, Xinhua Shen ${ }^{2}$ \\ ${ }^{1}$ School of Medicine, Southeast University, Nanjing, China; ${ }^{2}$ Department of Neurosis and Psychosomatic Diseases, Affiliated Huzhou Third \\ Municipal Hospital, Wenzhou Medical University, Huzhou Third Municipal Hospital, Huzhou, China; ${ }^{3}$ Department of Neurology, the Affiliated \\ Dongguan Hospital of Southern Medical University, Dongguan, China; ${ }^{4}$ Department of Neuropsychiatry, Affiliated Zhongda Hospital of Southeast \\ University, Nanjing, China \\ Contributions: (I) Conception and design: Y Yuan, X Shen; (II) Administrative support: Z Shen, X Hong; (III) Provision of study materials or patients: \\ Z Shen, L Ren; (IV) Collection and assembly of data: Z Shen, F Wang; (V) Data analysis and interpretation: L Cui; (VI) Manuscript writing: All \\ authors; (VII) Final approval of manuscript: All authors. \\ "These authors contributed equally to this work. \\ Correspondence to: Yonggui Yuan. Department of Psychiatry, Affiliated Zhongda Hospital of Southeast University, Nanjing, China. \\ Email: yygylh2000@sina.com; Xinhua Shen. Department of Neurosis and Psychosomatic Diseases, Huzhou Third Municipal Hospital, Huzhou, \\ China. Email: shenxinhuasun@sina.com.
}

Background: Generalized anxiety disorder (GAD) is partly attibuted to the dysregulation of nueroinflammation which can be mediated by adiponectin. We conducted this study was to explore the characteristics of peripheral adiponectin and its role in predicting treatment outcome in patients with generalized anxiety disorder (GAD) treated by escitalopram or venlafaxine.

Methods: A total of 70 untreated GAD inpatients who met the diagnosis criteria of the Diagnostic and Statistical Manual of Mental Disorders IV (DSM-IV) were enrolled and randomly selected for treatment with escitalopram $(n=36)$ or venlafaxine $(n=34)$ for 8 weeks. The serum adiponectin level of GAD and healthy controls (HCs) was measured by enzyme-linked immunosorbent assay (ELISA) before treatment. Hamilton Anxiety Rating Scale (HAM-A) assessment was conducted at baseline and at 1, 2, 4, and 8 weeks after treatment respectively. Serum adiponectin levels were compared between GAD patients and HCs, as well as between remission and nonremission cases; the correlation between baseline adiponectin level and HAM-A reduction rate were also analyzed.

Results: The serum adiponectin levels were higher in GAD patients compared to HCs $(t=2.304 ; \mathrm{P}=0.023)$, the serum adiponectin levels were higher in remission cases compared to nonremission cases $(t=2.255$, $\mathrm{P}=0.027$ ), and the receiver operating characteristic (ROC) area in predicting treatment remission was $0.652 \pm 0.066(\mathrm{P}=0.029)$. The correlation between baseline adiponectin level and HAM-A reduction rate of $\mathrm{GAD}$ cases treated with escitalopram and venlafaxine in the endpoint was $0.362(\mathrm{P}=0.030)$ and -0.026 $(\mathrm{P}=0.883)$, respectively, and the ROC area of baseline adiponectin level in predicting treatment remission was $0.72 \pm 0.086(\mathrm{P}=0.024)$ and $0.473 \pm 0.102(\mathrm{P}=0.469)$, respectively.

Conclusions: Peripheral adiponectin is upregulated in GAD, and it seems higher baseline adiponectin level predicts a better treatment remission treated by escitalopram but not with venlafaxine, which suggests adiponectin maybe a potential key biomarker in Chinese GAD.

Keywords: Generalized anxiety disorder (GAD); adiponectin; escitalopram; remission

Submitted Apr 22, 2021. Accepted for publication Jun 29, 2021.

doi: 10.21037/apm-21-1542

View this article at: https://dx.doi.org/10.21037/apm-21-1542 


\section{Introduction}

According to Alonso and Lépine [2007], anxiety disorders are the most prevalent psychiatric disorders, among which, generalized anxiety disorder (GAD) is one of the most common impairing anxiety disorders, with an estimated prevalence of $5.3 \%$ in urban China (1). GAD is defined as an excessive and uncontrollable anxiety lasting for at least 6 months, accompanied by somatic symptoms, such as restlessness, fatigue, difficulty in concentrating, irritability, muscle tension, and sleep disturbances (2). These symptoms can induce the clinically significant distress and impairment in various domains of functioning (2), which the individual cannot control. GAD has been reported to show a high comorbidity rate, which ranges from $40 \%$ to $98 \%$ particularly between GAD and major depression disorder (MDD) during the course of treatment (3-7). The typically high prevalence and similar effects of antidepressants imply that these two diseases may share a common neurobiological transmitter.

Adiponectin, a hormone produced by adipocytes, regulates the metabolic process and the neural pathway jointly with other organs, including the brain (8). Adiponectin is known to be capable of penetrating the blood-brain barrier (BBB) and modulating neuronal excitability and synaptic plasticity, thereby resulting in neural pathway remodeling (9). Gill et al. (10) discovered that adiponectin plays a significant role in inhibiting the proinflammatory factors, such as tumor necrosis factor alpha (TNF- $\alpha$ ) and interleuakin-6 (IL-6), and it may be positively correlated with mental disorders. Other studies have reported that exogenous injection of adiponectinneutralizing antibodies into the vein can alleviate anxiety and depression in the diabetic mouse model (11), indicating that adiponectin may be an essential transmitter of the anxiety neural pathway. In addition, Wilhelm et al. (12) demonstrated that the serum adiponectin level is positively correlated with anxiety in adults. Still other evidence shows that adiponectin insufficiency leads to aggravated anxiety behavior $(9,13)$. Therefore, we reasonably speculated that adiponectin might serve as an important biomarker in diagnosing anxiety. In the brain, the adiponectin signals rely on the receptors AdipoR1 and AdipoR2 to affect the important brain functions, including hippocampal neurogenesis and synaptic plasticity (14). Specifically, these two adiponectin receptors are found to be highly expressed in the brain areas related to anxiety disorder and depression, like the hippocampus and prefrontal cortex, where adiponectin acts as the antidepressant and antianxiety agent
$(11,15)$. Further, the injection of adiponectin or analogues into the ventral tegmental area of wild mice has been shown to reduce the activity and activation rate of basal dopamine neurons, suppress the activity of stress-induced dopamine neurons, and decrease anxiety behavior (13). These results have reinforced the idea that adiponectin is a vital biological factor in anxiety disorder.

Abundant evidence suggests that adiponectin may affect anxiety; there were several reports explored the predictive value of adiponectin in patients with anxiety disorder (14), but as to $\mathrm{GAD}$, the literature is limited, in fact anxiety disorders including many subtypes such as generalized anxiety disorder and panic disorder, few existing studies thus far have explored the association between adiponectin and GAD. Therefore, whether adiponectin has the same effect on GAD such as BDNF (brain derived neurotrophic factor), whether adiponectin changes with the alterations in GAD, and whether the baseline adiponectin level predicts the treatment response for GAD by different antidepressants remain unclear. To clarify these issues, this study had compared the adiponectin levels between healthy controls (HCs) and GAD patients to explore the differences between them. Additionally, the serum adiponectin levels were compared between anxiolytic remission and nonremission cases treated by escitalopram or venlafaxine, so as to examine the relationship of adiponectin with $\mathrm{GAD}$, and to further explore the role of adiponectin in prediction anxiolytic efficacy with different antidepressants. We present the following article in accordance with the STARD reporting checklist (available at https://dx.doi.org/10.21037/apm-21-1542).

\section{Methods}

\section{Participants}

A total of 180 patients were enrolled in this study that was carried out at the Huzhou Third Hospital; among these, 80 patients failed to meet the inclusion criteria, while 16 refused to participate in this study. Finally, 74 patients initially diagnosed with GAD in accordance with the Diagnostic and Statistical Manual of Mental Disorders IV (DSM-IV) criteria for GAD were included into this study. The patient inclusion criteria were as follows: patients aged 18-60 years; those with the scores of $>17$ on the Hamilton Anxiety Rating Scale (HAM-A) and $\leq 14$ on the 17 -item Hamilton Rating Scale for depression (HAM-D $\left.{ }_{17}\right)(16)$; and those with no neurological or related medical disorders, history of bipolar disorder, schizophrenia, or substance 
abuse or dependency (17). Before enrollment, all patients were free of any antidepressants and psychotherapy for at least 2 weeks. An additional 72 HCs were recruited from several communities and assessed by psychiatrists using the DSM-IV. The HAM-A scores of all controls were $\leq 7$. Moreover, HCs diagnosed with any psychiatric disorder were excluded. The study protocol was given approval by the Ethics Committee of Huzhou Third Municipal Hospital (No.: 2018-006). After a complete description of the research scheme, written informed consent was obtained from all participants before the study. All procedures performed in this study involving human participants were conducted in accordance with the Declaration of Helsinki (as revised in 2013).

\section{Treatment procedure}

All eligible patients were numbered according to the random number table method, and received escitalopram or venlafaxine with a flexible dosage randomly. No other antipsychotics or psychotherapy was allowed during the whole course of this study, and the raters were blinded to the medication. Specifically, 34 patients received venlafaxine (at a dose of 150-225 mg/d), while 36 received escitalopram (at a dose of 10-20 mg/d). Benzodiazepines were forbidden, while hypnotics such as zolpidem were administered for less than 2 weeks for insomnia if needed.

\section{Measurement of the adiponectin}

\section{Sample collection}

Blood samples were collected from 70 GAD patients at baseline (before treatment) and at 8 weeks after treatment. On the day or the next morning of blood collection, a $5 \mathrm{~mL}$ sample was collected from the separated rubber tube and centrifuged for 5 minutes at 3,500 rpm; then, the serum was separated and preserved within the cryopreservation tube at $-70{ }^{\circ} \mathrm{C}$. At the end of the 8 -week period, the blood sample was collected again and processed by the same method previously described. The $71 \mathrm{HC}$ samples were generally processed according to the same method as that of baseline.

\section{Determination of adiponectin}

The serum adiponectin level was determined through enzymelinked immunosorbent assay (ELISA) according to the double antibody sandwich method. The reagent was produced by Wuhan Boster Biotechnology Co, Ltd. All operations were carried out in accordance with related instructions.
To reduce the variation in measurement, the adiponectin level was measured after the collection of all blood samples.

\section{Clinical assessment}

The severity and efficacy of the GAD symptoms were rated with HAM-A in all patients by a single-blinded evaluator throughout the study. HAM-A score on week 8 was applied as the primary efficacy endpoint. HAM-A was assessed on baseline and at 1, 2, 4, and 8 weeks after treatment, while HAM-D was used to assess depression at corresponding time points. The HAM-A reduction rate was defined as follows: HAM-A reduction rate $=($ baseline HAM-A - endpoint HAM-A)/baseline HAM-A $\times 100 \%$; meanwhile, remission was defined as a HAM-A score $\leq 7$ at the endpoint of 8 weeks of treatment; otherwise, it was defined as nonremission (18). The evaluator was blinded to the biological data, whereas the laboratory workers were blinded to the HAM-A score, and both were blinded to our research purpose. A specialty data manager was assigned to keep all data and schedules.

\section{Statistical analysis}

The study endpoint for all analyses was week 8. SPSS 19.0 software (IBM Corp.) for Windows was adopted for data analysis. The normally distributed data are expressed as mean \pm SD. Student's $t$-tests were used to compare the difference in demographic and adiponectin data, including the adiponectin levels in GAD and HCs, as well as between remission and nonremission cases. Chi-square test was used for frequency comparison. The association between variables and the adiponectin level was explored through Pearson correlation. Receiver operating characteristic curve (ROC) was applied to explore the predictive value of baseline adiponectin level in treatment remission. A 2-tailed $\mathrm{P}$ value $<0.05$ was considered to be statistically significant.

\section{Results}

\section{Demographical characteristics and adiponectin levels of $G A D$ patients and $H C$}

We failed to acquire the adiponectin results in $4 \mathrm{GAD}$ patients and one HC, so the data of 70 GAD cases and $71 \mathrm{HCs}$ were analyzed at the study endpoint. There was no significant difference in the male:female ratio, age, education level, or BMI between GAD patients and HCs $(\mathrm{P}>0.05)$, but the positive family rates in GAD were 
Table 1 Demographical and clinical characteristics of GAD and HC

\begin{tabular}{|c|c|c|c|c|}
\hline Characteristics & GAD $(n=70)$ & $\mathrm{HC}(\mathrm{n}=71)$ & \multicolumn{2}{|c|}{ Statistics } \\
\hline Age & $45.7 \pm 9.6$ & $42.5 \pm 10.5$ & 1.886 & 0.061 \\
\hline Sex (male/female) & $13 / 57$ & $15 / 56$ & 0.145 & 0.704 \\
\hline Education (years) & $7.3 \pm 4.0$ & $7.9 \pm 4.3$ & 0.815 & 0.417 \\
\hline Family history (yes/no) & $13 / 57$ & $3 / 68$ & 5.413 & 0.020 \\
\hline Adiponectin $(\mu \mathrm{g} / \mathrm{mL})$ & $3.94 \pm 1.98$ & $2.97 \pm 1.57$ & 2.255 & 0.027 \\
\hline
\end{tabular}

GAD, generalized anxiety disorder; $\mathrm{HC}$, healthy control.

Table 2 Clinical characteristics and adiponectin status of the remission and nonremission group

\begin{tabular}{|c|c|c|c|c|}
\hline Characteristics & Remission $(\mathrm{n}=36)$ & Nonremission $(n=34)$ & \multicolumn{2}{|c|}{ Statistics } \\
\hline Age & $46.0 \pm 9.5$ & $45.4 \pm 9.9$ & 0.266 & 0.791 \\
\hline Sex (male/female) & $6 / 30$ & $7 / 27$ & 0.178 & 0.673 \\
\hline BMI $\left(\mathrm{kg} / \mathrm{m}^{2}\right)$ & $22.1 \pm 2.7$ & $21.5 \pm 2.3$ & 0.892 & 0.376 \\
\hline Family history (yes/no) & $7 / 29$ & $6 / 28$ & 0.037 & 0.847 \\
\hline Baseline HAM-A & $20.8 \pm 3.3$ & $22.6 \pm 2.9$ & 2.343 & 0.022 \\
\hline Baseline HAM-D & $9.8 \pm 2.6$ & $11.1 \pm 2.1$ & 2.324 & 0.023 \\
\hline 8-week HAM-A & $4.4 \pm 1.7$ & $12.4 \pm 3.8$ & 11.436 & $<0.001$ \\
\hline
\end{tabular}

HAM-A, Hamilton Anxiety Rating Scale; HAM-D, Hamilton Rating Scale for depression.

significantly higher than those in $\mathrm{HCs}\left(\chi^{2}=5.413 ; \mathrm{P}=0.020\right)$.

The serum adiponectin levels in GAD patients were higher compared to those in HCs $(t=2.304 ; \mathrm{P}=0.023$; for details see Table 1). The serum adiponectin levels in GAD patients had no correlation with age $(\mathrm{r}=0.038, \mathrm{P}=0.756)$, baseline HAM-A scores $(r=-0.147 ; \mathrm{P}=0.224)$, baseline HAMD scores $(\mathrm{r}=-0.105 ; \mathrm{P}=0.389)$, or illness duration $(\mathrm{r}=-0.092 ; \mathrm{P}=0.419)$, and there were no significant differences in adiponectin levels between male $(3.47 \pm 1.84 \mathrm{ug} / \mathrm{mL})$ and female $(3.47 \pm 1.84 \mathrm{ug} / \mathrm{mL})$ GAD cases $(t=2.304 ; \mathrm{P}=0.023)$.

\section{Comparison of clinical characteristics and adiponectin levels in the remission group and remission group}

Ultimately, 36 participants achieved remission, and 34 did not achieve remission. Differences in age, gender, BMI, illness duration, and family history were not statistically significant between the remission and nonremission groups $(\mathrm{P}>0.05)$. Meanwhile, baseline HAM-A and HAM-D scores were statistically lower in the remission group than in the nonremission group $(\mathrm{P}<0.05$; for details see Table 2$)$.

The serum adiponectin levels were higher in remission cases $(3.94 \pm 1.98 \mathrm{ug} / \mathrm{mL})$ compared to nonremission cases $(2.97 \pm 1.57 \mathrm{ug} / \mathrm{mL} ; t=2.255 ; \mathrm{P}=0.027)$.

\section{The roles of baseline adiponectin in predicting treatment outcome in GAD patients}

The HAM-A reduction rates after 1, 2, 4, and 8 weeks of 
treatment were $18.6 \% \pm 10.5 \%, 35.6 \% \pm 16.5 \%, 53.0 \% \pm 19.1 \%$, and $62.2 \% \pm 21.0 \%$, respectively, all of which were positively correlated with baseline adiponectin level $(\mathrm{r}=0.330, \mathrm{P}=0.005$;

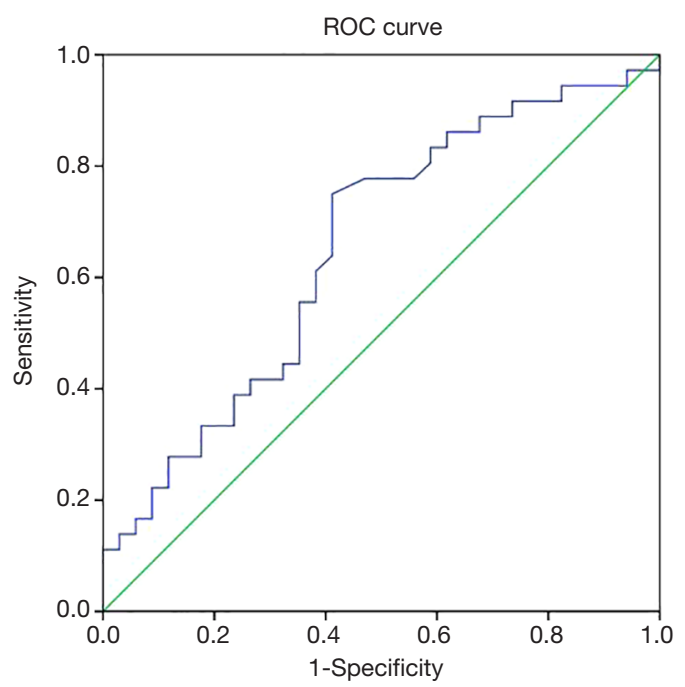

Figure 1 ROC curve of adiponectin level in the diagnosis of GAD. ROC, receiver operating characteristic; GAD, generalized anxiety disorder. $\mathrm{r}=0.252, \mathrm{P}=0.035 ; \mathrm{r}=0.272, \mathrm{P}=0.023 ; \mathrm{r}=0.248, \mathrm{P}=0.039)$. In GAD patients, the ROC area of baseline adiponectin level in predicting treatment remission at the endpoint ( 8 weeks) was $0.652 \pm 0.066(\mathrm{P}=0.029$; Figure 1$)$.

The data of $36 \mathrm{GAD}$ patients treated with escitalopram and 34 cases treated with venlafaxine were analyzed. Differences in age, gender, BMI, illness duration, family history, baseline HAM-A and HAM-D scores were not statistically significant between these treatment groups ( $\mathrm{P}>0.05)$, nor was the HAM-A reduction rate after 1, 2, 4, and 8 weeks of treatment $(\mathrm{P}>0.05$; for details see Table 3$)$.

The correlation between baseline adiponectin level and HAM-A reduction rate of GAD cases treated with escitalopram at endpoint was 0.362 ( $\mathrm{P}=0.030$; Figure 2), and the ROC area of baseline adiponectin level in predicting treatment remission treated with escitalopram in the endpoint was $0.722 \pm 0.086(\mathrm{P}=0.024$; Figure 3). The correlation between baseline adiponectin level and HAM-A reduction rate of $G A D$ cases treated with venlafaxine at endpoint was $-0.026(\mathrm{P}=0.883$; Figure 4$)$, and the $\mathrm{ROC}$ area of baseline adiponectin level in predicting treatment remission in the endpoint was $0.473 \pm 0.102(\mathrm{P}=0.469$; Figure 5).

Table 3 Clinical characteristics and adiponectin of GAD cases treated with escitalopram or venlafaxine

\begin{tabular}{|c|c|c|c|c|}
\hline Characteristics & Escitalopram ( $\mathrm{n}=36)$ & Venlafaxine $(n=34)$ & \multicolumn{2}{|c|}{ Statistics } \\
\hline Age & $44.7 \pm 10.3$ & $46.8 \pm 8.9$ & 0.899 & 0.372 \\
\hline Sex (male/female) & $7 / 29$ & $6 / 28$ & 0.037 & 0.847 \\
\hline BMI $\left(\mathrm{kg} / \mathrm{m}^{2}\right)$ & $21.6 \pm 2.6$ & $22.1 \pm 2.5$ & 0.779 & 0.438 \\
\hline Family history (yes/no) & $7 / 29$ & $6 / 28$ & 0.037 & 0.847 \\
\hline Baseline HAM-A & $20.9 \pm 3.1$ & $22.4 \pm 3.3$ & 1.927 & 0.057 \\
\hline Baseline HAMD & $10.1 \pm 2.2$ & $10.9 \pm 2.7$ & 1.378 & 0.173 \\
\hline Adiponectin ( $\mu \mathrm{g} / \mathrm{mL})$ & $3.6 \pm 2.1$ & $3.3 \pm 1.6$ & 0.615 & 0.541 \\
\hline 4-w HAM-A reduction rate (\%) & $56.7 \pm 22.6$ & $49.0 \pm 13.8$ & 1.722 & 0.090 \\
\hline 8-w HAM-A reduction rate (\%) & $63.9 \pm 24.4$ & $60.5 \pm 16.6$ & 0.672 & 0.500 \\
\hline Remission/nonremission & $20 / 16$ & $16 / 18$ & 0.505 & 0.477 \\
\hline Medication dose (mg/d) & $16.2 \pm 4.1$ & $151.0 \pm 44.4$ & NA & NA \\
\hline
\end{tabular}

GAD, generalized anxiety disorder; HAM-A, Hamilton Anxiety Rating Scale; HAM-D, Hamilton Rating Scale for depression. 


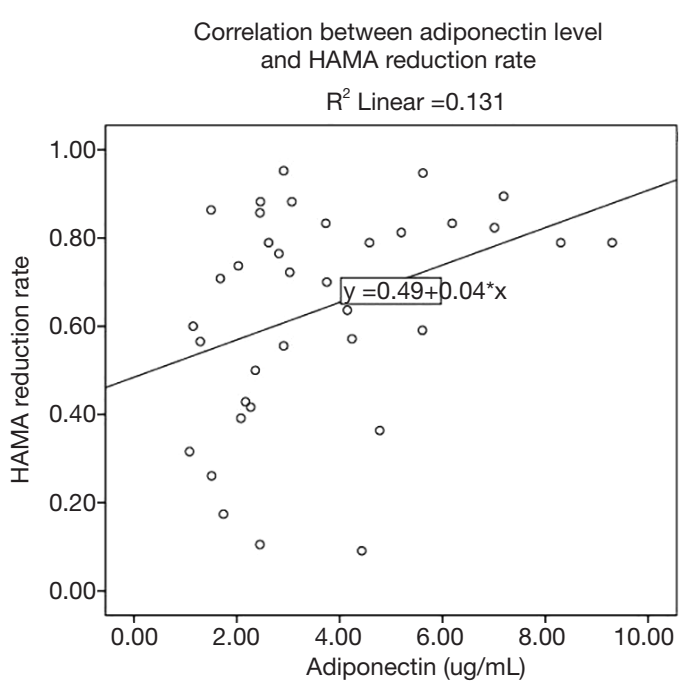

Figure 2 Correlation between baseline adiponectin level and HAM-A reduction rate with escitalopram treatment. HAM-A, Hamilton Anxiety Rating Scale.

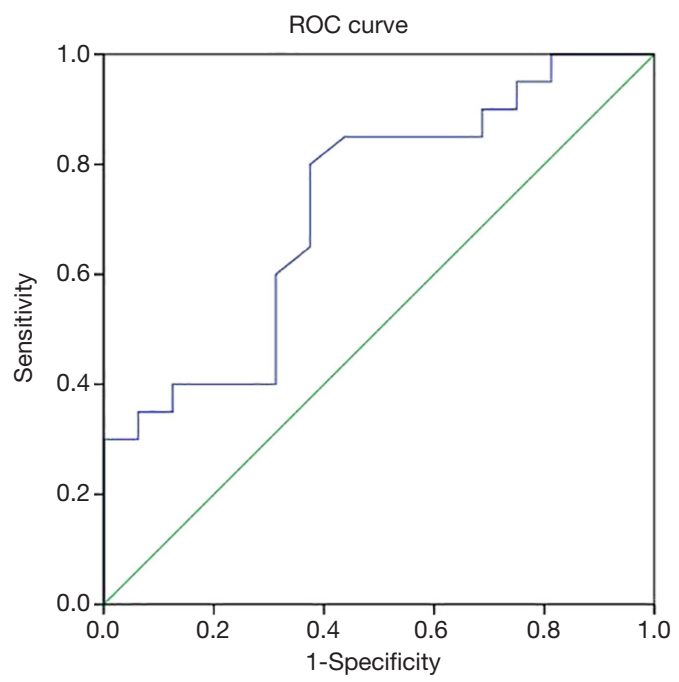

Figure 3 ROC curve of baseline adiponectin level in predicting remission of patients treated by escitalopram. ROC, receiver operating characteristic.

\section{Discussion}

This study aimed to explore the relationship of serum adiponectin with GAD, together with the characteristics of adiponectin level in GAD patients and its role in predicting treatment remission with different antidepressants. The principal results of this study were the following: (I) the serum adiponectin levels were upregulated in GAD patients;

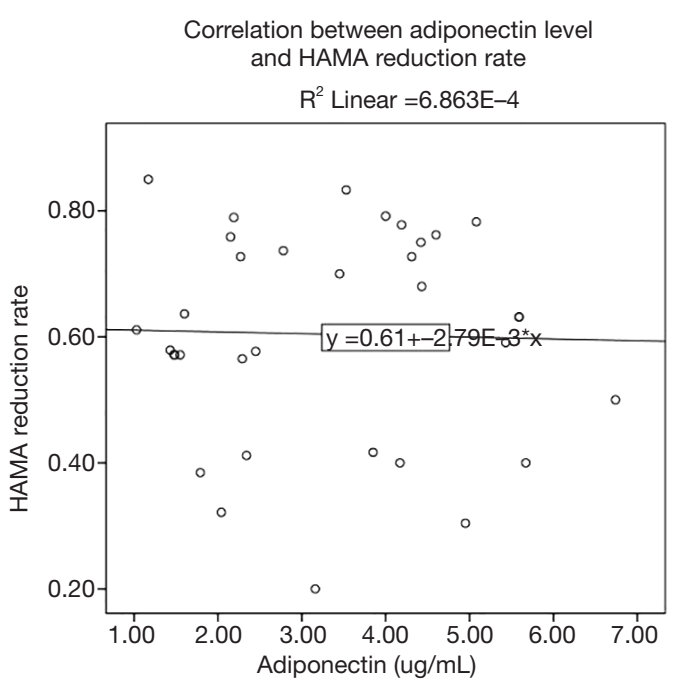

Figure 4 Correlation between baseline adiponectin level and HAM-A reduction rate in patients treated with venlafaxine. HAM-A, Hamilton Anxiety Rating Scale.

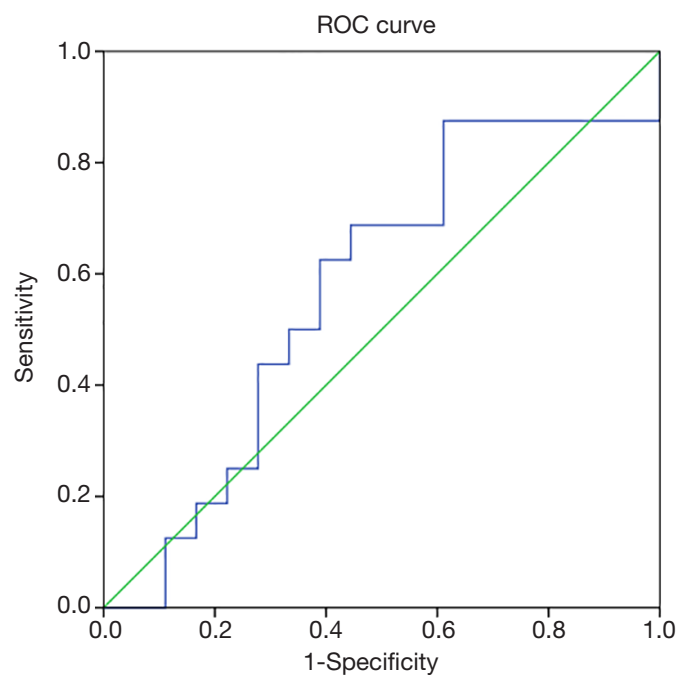

Figure 5 ROC curve of baseline adiponectin level in predicting remission of patients treated by venlafaxine. ROC, receiver operating characteristic.

the serum adiponectin levels in GAD patients had no correlation with age, anxiety severity and illness duration; and there were no adiponectin level differences according to gender in GAD patients. (II) The serum adiponectin levels were higher in remission cases compared to nonremission cases. (III) The HAM-A reduction rates throughout the study were positively correlated with baseline adiponectin 
level, which means a higher baseline adiponectin level may predict a higher anxiety syndrome improvement in GAD patients; meanwhile, the ROC area of baseline adiponectin level had good value in predicting treatment remission in the acute treatment stage of GAD patients treated with escitalopram, but had no value in predicting outcome treated with venlafaxine.

Adiponectin, a metabolic cytokine which is secreted by adipocytes, is involved in stress-related disorders, including anxiety disorder and depression. It can cross the BBB to act on neurons in the central nervous system with insulinsensitizing, anti-inflammatory, and antiatherogenic effects (19-21). Meanwhile, the peripheral adiponectin level can reflect the change of adiponectin in the brain (19-21). To date, many studies have focused on the relationship of MDD with adiponectin, but these have produced inconsistent results (19-21). Regarding anxiety, several studies have shown that the deficiency of adiponectin increases the susceptibility to social defeat stress $(11,22,23)$, suggesting that adiponectin may serve as an important biomarker for stress-related disorders and mood disorder; however, the pathological mechanism responsible for this has not been completely elucidated.

It is possible that this mechanism may work as follows: long-term social defeat stress activates the sympathetic nervous system, and the sympathetic nerve fibers directly govern the white adipose tissue and release norepinephrine at the neuro-adipose junctions (24), which may lead to dysregulated adiponectin level, causing anxiety. It was reported that the intra-ventral tegmental area (VTA) infusion of adiponectin caused anxiety behavior in mice but was abolished in mice lacking AdipoR1 in the dopamine neurons (19-21). As suggested in our study, the adiponectin levels in GAD patients were upregulated. Studies focusing on the effect of adiponectin on GAD are scarce. Wagner et al reported GAD patients had lower adiponectin compared to HCs, but they also found decreased interleukin 6 (IL-6) levels in GAD patients-a fact which conflicts with the hypothesis that GAD involves mild inflammation (19-21). Another study found that adiponectin levels were lower in a panic disorder patient group compared to HCs (14). Such contradictory results might be attributed to the following: (I) physical exercise mediates adiponectin level, consistent with the results from Yau et al. (23). More specifically, physical exercise facilitates adiponectin production (25), and the particular symptoms of GAD can be alleviated after physical activity exposure (26). Therefore, physical exercise may increase the adiponectin level and in turn mitigate anxiety.
(II) Drugs may also elevate the endogenous adiponectin level. Some evidence shows that the adiponectin level may increase after antidepressant treatment (27). GAD is a longlasting anxiety disorder, and in our study, many samples suffered from multiple relapses and received antianxiety medications for a prolonged period. Therefore, it may be reasonably inferred that the cumulative effect of longterm medication may result in an increase in adiponectin levels among GAD patients. (III) Adiponectin levels can be affected by sex, age, anxiety subtype, blood sample type, fasting state, blood sample collection time, and the assay used. The findings from this study suggest that the serum adiponectin had no correlation with age, HAM-A score, HAMD score, or illness duration levels in GAD patients. Furthermore, there were no significant differences found between male and female GAD patients, which is partly consistent with the conclusion drawn by Formolo et al. (7).

As shown by our results, the adiponectin level was higher in the remission group than in the nonremission group, and the ROC curve of baseline adiponectin level also suggested a predictive value of adiponectin level in the treatmentinduced remission of GAD. Interestingly, the ROC area of baseline adiponectin level had a good value in predicting the treatment remission of escitalopram but not that of venlafaxine. There have been results showing that disorders in the proinflammatory and anti-inflammatory responses can lead to abnormal cytokine levels among GAD patients (28). Inflammatory factors can activate the hypothalamicpituitary-adrenal axis, which thereby activates the $5-\mathrm{HT}$ transporter, causing a reduction in the serotonin available in the brain (29). Escitalopram is a selective serotonin reuptake inhibitor (SSRI) which acts on the 5-HT system; meanwhile, venlafaxine is a serotonin norepinephrine reuptake inhibitor (SNRI) acting on 5-HT and Norepinephrine systems; both are recommended as a firstline anxiolytic drugs (28). Adiponectin plays a vital role in inhibiting inflammation, especially for TNF- $\alpha$. Additionally, adiponectin represses the production of TNF- $\alpha$ while simultaneously inducing the production of various antiinflammatory factors (30). As a result, the adiponectin level is negatively correlated with TNF- $\alpha$. Hou et al. (31) discovered that patients who achieved greater reduction in anxiety had greater decreases in the cytokine levels, which can be partly explained by the inhibiting inflammation role of adiponectin. Consequently, higher baseline adiponectin levels may predict a better treatment outcome in GAD patients treated by escitalopram.

Inheritance should also be given its due attention in this 
discussion. It has been reported that genes contribute to 30 $50 \%$ of the emergence of anxiety disorders $(32,33)$, whereas the prevalence of nongenetic anxiety disorders is about $50-70 \%$. On the other hand, environmental factors may affect the development of anxiety through heredity. Even the mother of a child may receive an anxiety-susceptibility factor during pregnancy, whose effects may be exacerbated by environmental conditions (34). For instance, for mothers who are diagnosed with anxiety and who are not receiving medication, DNA methylation alteration in the promoter region of the glucocorticoid receptor gene can be found in the umbilical cord blood and genome $(35,36)$, which may increase the risk of anxiety in the child. Therefore, genetics exerts a significant influence on development, which is consistent with our results. This study observed a statistical difference in family history, and there were more patients with a family history in the GAD group than in the HC group . However, the family history showed no significant difference between the remission and nonremission groups. Anxiolytic drugs maybe a factor in the treatment outcome which effect the predictive value of adiponectin in the treatment of GAD; however, the GAD patients in our study, regardless of being treated with escitalopram or venlafaxine, expressed a similar HAM-A reduction rate.

Some limitations should be noted in this study. The sample size employed was too small to prove that adiponectin can serve as a predictive biomarker. Nonetheless, the adiponectin hormone is still considered to have potential as a antianxiety and antidepressant supplement. In addition, a larger sample size may potentially reveal the significant difference in adiponectin level between GAD patients and HCs or between the remission and nonremission groups. Another limitation was that this was a case-control study, which only revealed the association between adiponectin level and remission. As a result, more detailed longitudinal studies should be designed to explain the causal relationship and the change of adiponectin level throughout the treatment. In the meantime, the relationship between adiponectin level and GAD should also be explored based on controlling the covariates (e.g., age, BMI, exercise, and drugs) (3). Further studies are warranted to examine the potential mechanism of the adiponectin effect on GAD.

In summary, our results indicate that serum adiponectin was upregulated in GAD patients and that a higher baseline adiponectin level predicted greater chance of remission in patients treated by escitalopram. Therefore, adiponectin may be a key biomarker in GAD and worthy of further investigation. Variables such as duration of illness, patient selection, illness subtype, assessment tool and cutoff scores employed, comorbid illness, and psychotropic treatment should all be considered when interpreting findings from studies of adiponectin, as they all have been shown to affect circulating levels.

\section{Acknowledgments}

Funding: This study was partly supported by funds from the Huzhou Public Welfare Research Project Social Development (Key Medical and Health) Category (No. 2018GZ39 to Xinhua Shen), Huzhou Public Welfare Research Project Social Development Category (2018GYB49, Zhongxia Shen), the Social Development Project of Public Welfare Technology Application in Zhejiang Province in 2019 (No. LGF19H090003 to Xinhua Shen), and the Social Development Project of Public Welfare Technology Application in Zhejiang Province in 2019 (No. LGF19H090002 to Zhongxia Shen). We would like to express our heartfelt thanks to all participants who contributed to this study.

\section{Footnote}

Reporting Checklist: The authors have completed the STARD reporting checklist. Available at https://dx.doi. org/10.21037/apm-21-1542

Data Sharing Statement: Available at https://dx.doi. org/10.21037/apm-21-1542

Conflicts of Interest: All authors have completed the ICMJE uniform disclosure form (available at https://dx.doi. org/10.21037/apm-21-1542). The authors reported that this study was partly supported by funds from the Huzhou Public Welfare Research Project Social Development (Key Medical and Health) Category (No. 2018GZ39 to XS), Huzhou Public Welfare Research Project Social Development Category (2018GYB49, ZS), the Social Development Project of Public Welfare Technology Application in Zhejiang Province in 2019 (No. LGF19H090003 to XS), and the Social Development Project of Public Welfare Technology Application in Zhejiang Province in 2019 (No. LGF19H090002 to ZS). The authors have no other conflicts of interest to declare.

Ethical Statement: The authors are accountable for all aspects of the work in ensuring that questions related 
to the accuracy or integrity of any part of the work are appropriately investigated and resolved. The study protocol was given approval by the Ethics Committee of Huzhou Third Municipal Hospital (No.: 2018-006). After a complete description of the research scheme, written informed consent was obtained from all participants before the study. All procedures performed in this study involving human participants were conducted in accordance with the Declaration of Helsinki (as revised in 2013).

Open Access Statement: This is an Open Access article distributed in accordance with the Creative Commons Attribution-NonCommercial-NoDerivs 4.0 International License (CC BY-NC-ND 4.0), which permits the noncommercial replication and distribution of the article with the strict proviso that no changes or edits are made and the original work is properly cited (including links to both the formal publication through the relevant DOI and the license). See: https://creativecommons.org/licenses/by-nc-nd/4.0/.

\section{References}

1. Yu W, Singh SS, Calhoun S, et al. Generalized anxiety disorder in urban China: Prevalence, awareness, and disease burden. J Affect Disord 2018;234:89.

2. Association AP. Diagnostic and statistical manual of mental disorders. 4th. ed. Washington, DC; 2000.

3. Newman MG, Amy P, Fisher AJ, et al. Diagnostic comorbidity in adults with generalized anxiety disorder: impact of comorbidity on psychotherapy outcome and impact of psychotherapy on comorbid diagnoses. Behav Ther 2010;41:59-72.

4. Hung CI, Liu CY, Yang CH, et al. Comorbidity with more anxiety disorders associated with a poorer prognosis persisting at the 10-year follow-up among patients with major depressive disorder. J Affect Disord 2020;260:97-104.

5. Goisman RM, Goldenberg I, Vasile RG, et al. Comorbidity of anxiety disorders in a multicenter anxiety study. Compr Psychiatry 1995;36:303-11.

6. Shen Z, Zhu J, Ren L, et al. Aberrant amplitude lowfrequency fluctuation (ALFF) and regional homogeneity (ReHo) in generalized anxiety disorder (GAD) and their roles in predicting treatment remission. Ann Transl Med 2020;8:1319.

7. Formolo DA, Lee TH, Yau SY. Increasing Adiponergic System Activity as a Potential Treatment for Depressive Disorders. Mol Neurobiol 2019;56:7966-76.

8. Guo M, Li C, Lei Y, et al. Role of the adipose
PPAR $\gamma$-adiponectin axis in susceptibility to stress and depression/anxiety-related behaviors. Mol Psychiatry 2017;22:1056-68.

9. Zhang D, Guo M, Zhang W, et al. Adiponectin stimulates proliferation of adult hippocampal neural stem/progenitor cells through activation of $\mathrm{p} 38$ mitogen-activated protein kinase (p38MAPK)/glycogen synthase kinase $3 \beta$ (GSK-3 $\beta$ )/ $\beta$-catenin signaling cascade. J Biol Chem 2011;286:44913.

10. Gill J, Vythilingam M, Page GG. Low cortisol, high DHEA, and high levels of stimulated TNF-alpha, and IL-6 in women with PTSD. J Trauma Stress 2008;21:530-9.

11. Liu J, Guo M, Zhang D, et al. Adiponectin is critical in determining susceptibility to depressive behaviors and has antidepressant-like activity. Proc Natl Acad Sci U S A 2012;109:12248-53.

12. Wilhelm CJ, Dongseok C, Marilyn H, et al. Adipocytokine signaling is altered in Flinders sensitive line rats, and adiponectin correlates in humans with some symptoms of depression. Pharmacol Biochem Behav 2013;103:643.

13. Sun F, Lei Y, You J, et al. Adiponectin modulates ventral tegmental area dopamine neuron activity and anxietyrelated behavior through AdipoR1. Mol Psychiatry 2019;24:126-44.

14. Vuong E, Nothling J, Lombard C, et al. Peripheral adiponectin levels in anxiety, mood, trauma- and stressorrelated disorders: A systematic review and meta-analysis. J Affect Disord 2020;260:372-409.

15. Zhang D, Wang X, Lu XY. Adiponectin exerts neurotrophic effects on dendritic arborization, spinogenesis and neurogenesis in the dentate gyrus in male mice. Endocrinology 2016;157:2853-69.

16. Qian M, Shen Z, Lin M. Early improvement predicts 8 -week treatment outcome in patients with generalized anxiety disorder treated with escitalopram or venlafaxine. Asia Pac Psychiatry 2017;9:e12270.

17. Weber-Hamann B, Kratzsch J, Kopf D, et al. Resistin and adiponectin in major depression: The association with free cortisol and effects of antidepressant treatment. J Psychiatr Res 2007;41:344-50.

18. Shen XH, Qian MC, Niu FR, et al. Dynamic level observation of brain-derived neurotrophic factor in patients with first-episode generalized anxiety disorder. Zhonghua Yi Xue Za Zhi 2011;91:2939-41.

19. Jow GM, Yang TT, Chen CL. Leptin and cholesterol levels are low in major depressive disorder, but high in schizophrenia. J Affect Disord 2006;90:21-7. 
20. Cizza G, Nguyen VT, Eskandari F, et al. Low 24-hour adiponectin and high nocturnal leptin concentrations in a case-control study of community-dwelling premenopausal women with major depressive disorder: the Premenopausal, Osteopenia/Osteoporosis, Women, Alendronate, Depression (POWER) study. J Clin Psychiatry 2010;71:1079-87.

21. Kotan Z, Sarandöl E, Kurhan E, et al. Serum brain-derived neurotrophic factor, vascular endothelial growth factor and leptin levels in patients with a diagnosis of severe major depressive disorder with melancholic features. Ther Adv Psychopharmacol 2012;2:65-74.

22. Zhang D, Wang X, Wang B, et al. Adiponectin regulates contextual fear extinction and intrinsic excitability of dentate gyrus granule neurons through AdipoR2 receptors. Mol Psychiatry 2017;22:1044-55.

23. Yau SY, Li A, Hoo RL, et al. Physical exercise-induced hippocampal neurogenesis and antidepressant effects are mediated by the adipocyte hormone adiponectin. Proc Natl Acad Sci U S A 2014;111:15810.

24. Bowers RR, Festuccia WT, Song CK, et al. Sympathetic innervation of white adipose tissue and its regulation of fat cell number. Am J Physiol Regul Integr Comp Physiol 2004;286:R1167-75.

25. Vu V, Riddell MC, Sweeney G. Circulating adiponectin and adiponectin receptor expression in skeletal muscle: effects of exercise. Diabetes Metab Res Rev 2007;23:600-11.

26. McDowell CP, Dishman RK, Gordon BR, et al. Physical Activity and Anxiety: A Systematic Review and Metaanalysis of Prospective Cohort Studies. Am J Prev Med 2019;57:545-56.

27. Narita K, Murata T, Takahashi T, et al. Plasma levels of adiponectin and tumor necrosis factor-alpha in patients with remitted major depression receiving long-term maintenance antidepressant therapy. Prog

Cite this article as: Shen Z, Wang F, Cui L, Ren L, Hong X, Yuan Y, Shen X. Higher baseline serum adiponectin predicts better treatment remission in patients with generalized anxiety disorder treated with escitalopram. Ann Palliat Med 2021;10(7):7634-7643. doi: 10.21037/apm-21-1542
Neuropsychopharmacol Biol Psychiatry 2006;30:1159-62.

28. Hou R, Garner M, Holmes C, et al. Peripheral inflammatory cytokines and immune balance in Generalised Anxiety Disorder: Case-controlled study. Brain Behav Immun 2017;62:212-8.

29. Berthold-Losleben M, Himmerich H. The TNF-alpha system: functional aspects in depression, narcolepsy and psychopharmacology. Curr Neuropharmacol 2008;6:193-202.

30. Tilg H, Wolf AM. Adiponectin: a key fat-derived molecule regulating inflammation. Expert Opin Ther Targets 2005;9:245-51.

31. Hou R, Ye G, Liu Y, et al. Effects of SSRIs on peripheral inflammatory cytokines in patients with Generalized Anxiety Disorder. Brain Behav Immun 2019;81:105-10.

32. Hettema JM, Neale MC, Kendler KS. A review and metaanalysis of the genetic epidemiology of anxiety disorders. Am J Psychiatry 2001;158:1568-78.

33. Smoller JW, Block SR, Young MM. Genetics of anxiety disorders: the complex road from DSM to DNA. Depress Anxiety 2009;26:965-75.

34. Patriquin MA, Mathew SJ. The Neurobiological Mechanisms of Generalized Anxiety Disorder and Chronic Stress. Chronic Stress (Thousand Oaks) 2017;1:247054701770399.

35. Hompes T, Izzi B, Gellens E, et al. Investigating the influence of maternal cortisol and emotional state during pregnancy on the DNA methylation status of the glucocorticoid receptor gene $(\mathrm{NR} 3 \mathrm{C} 1)$ promoter region in cord blood. J Psychiatr Res 2013;47:880-91.

36. Non AL, Binder AM, Kubzansky LD, et al. Genomewide DNA methylation in neonates exposed to maternal depression, anxiety, or SSRI medication during pregnancy. Epigenetics 2014;9:964-72.

(English Language Editor: J. Gray) 